\section{LA APORTACIÓN DE LA DEFENSA A LA MARCA ESPAÑA}

\author{
Mạ del Mar Hidalgo García \\ Analista del Instituto Español de Estudios Estratégicos \\ mhidga1@oc.mde.es
}

\section{DEFENCE CONTRIBUTION TO "BRAND SPAIN"}

Cómo citar este artículo/Citation: Hidalgo García, M. M. (2014). "La aportación de la defensa a la Marca España". Arbor, 190 (765): a102. doi: http://dx.doi.org/10.3989/ arbor.2014.765n1009

Recibido: 5 junio 2012. Aceptado: 2 diciembre 2013.

RESUMEN: En plena crisis económica se ha lanzado el proyecto "Marca España" como una política de estado para poner en valor el concepto de España y respaldar su proyección internacional. Bajo esta perspectiva la defensa tiene mucho que aportar a este reconocimiento internacional. En el artículo se exponen tres aspectos, donde España ejerce una gran influencia en el exterior, en términos de reputación, innovación y compromiso: la incorporación de la mujer a las FAS, las misiones en el exterior y la industria de defensa.

PALABRAS CLAVE: Marca España; Fuerzas Armadas; industria de defensa; misiones en el exterior; mujer militar.
Copyright: (C) 2014 CSIC. Este es un artículo de acceso abierto distribuido bajo los términos de la licencia Creative Commons Attribution-Non Commercial (by-nc) Spain 3.0.

ABSTRACT: The "Brand Spain" project was launched in the midst of the crisis as a government policy to boost Spain's image and raise its international profile. Defence has much to contribute to this international recognition. This paper describes three aspects, where Spain performs a great influence in terms of reputation, innovation and commitment: women joining the armed forces, international missions, and the defence industry.

KEYWORDS: Brand Spain; Armed Forces; defence industry; international missions; military women. 


\section{LA MARCA ESPAÑA}

En plena época de crisis mundial, en la que España está inmersa sufriendo sus duras consecuencias, es necesario levantar la vista y mirar al exterior para ver qué oportunidades se nos ofrecen fuera de nuestras fronteras que puedan contribuir a nuestro desarrollo económico. La aparición de potencias emergentes abre nuevos mercados con grandes posibilidades para la expansión internacional de muestras empresas.

Uno de los pilares sobre los que debe sustentarse la recuperación de nuestro país debe estar constituido por alcanzar una reputación y ganarse la confianza en un mundo complejo y globalizado y que cambia a una velocidad de vértigo. En este contexto, España necesita proyectar una imagen que vaya mucho más allá de la promoción turística de sol, playa y gastronomía que, si bien es muy importante pues supone un motor de crecimiento para la economía nacional, ya es bien conocida fuera de nuestras fronteras. La imagen debe trasmitir modernidad, seriedad, compromiso con sus socios y aliados, profesionalidad e innovación tecnológica, todo ello sin olvidar nuestra esencia cultural e histórica. Bajo esta perspectiva, se ha lanzado el proyecto "Marca España" como una política de estado para poner en valor el concepto de España.

Llevar a cabo esta tarea no es fácil. Como en cualquier lanzamiento de una marca, es necesario definir los atributos y su posicionamiento en el mercado de un producto que es intangible, único en concepto pero compuesto de múltiples factores, con un ciclo de vida en la que la fase de declive no debe producirse nunca. El mercado donde se lanza la marca está caracterizado por ser altamente competitivo en donde alcanzar una posición estratégica es una tarea, si no es imposible, si muy complicada. Se trata de ofrecer y de atraer, de vender pero a la vez ser considerado un buen cliente.

Son muchos los elementos que contribuyen a la marca España: económicos, políticos, culturales, tecnológicos, sociales y deportivos. Todos y cada uno de ellos pueden contribuir a promocionar nuestro país. En la presentación del portal creado a tal fin (http:// marcaespana.es) lo especifica con bastante claridad: "Una buena imagen-país es un activo que sirve para respaldar la posición internacional de un Estado política, económica, cultural, social, científica y tecnológicamente".

Una vez centrada la necesidad de realizar esta promoción, hay que establecer los elementos que pueden ser más eficaces para conseguir el objetivo. Entre ellos, y teniendo en cuenta la temática de este número, habría que destacar los que hacen referencia a las Fuerzas Armadas, como institución encargada de la defensa nacional y del mantenimiento del compromiso de España con la paz y la seguridad internacional dentro de las organizaciones internacionales de las que forma parte.

\section{LA DEFENSA COMO PROMOCIÓN DE LA MARCA ESPAÑA}

Las nuevas amenazas a la seguridad a nuestra seguridad y el compromiso con nuestros socios y aliados han propiciado que, a lo largo de las últimas décadas, las Fuerzas Armadas españolas se hayan visto sometidas a unos cambios, tanto en su organización como en sus funciones, necesarios para afrontar los nuevos retos dentro y fuera de nuestras fronteras.

Según el IX Estudio CIS sobre las Fuerzas Armadas y Sociedad, la población española considera que la misión principal de las Fuerzas Armadas es garantizar la soberanía y la integridad territorial y que además su actuación contribuye en gran medida al prestigio internacional de un país ${ }^{1}$. Por otro lado, en el mismo estudio se recoge que alrededor de un $80 \%$ de la población española aprueba mayoritariamente la participación de las FAS en el exterior. Esta cifra permanece casi invariable si se compara con los resultados de años anteriores, lo que demuestra que la sociedad ya se ha acostumbrado a la proyección exterior de nuestras FAS.

Volviendo al planteamiento inicial de poner en valor la imagen España, es justo reconocer cómo esta acción de las FAS, dentro y fuera de nuestras fronteras, proyecta una imagen que aporta seriedad, fiabilidad, modernidad y compromiso con la comunidad internacional. Si hubiera que seleccionar una institución en España en la que se han producido más cambios y que mejor ha sabido digerirlos para adaptarse a las circunstancias actuales, una candidatura seria, sería es sin duda, la de las Fuerzas Armadas.

A continuación se detallaran algunos ejemplos, que merece la pena destacar, para poner de manifiesto cómo las Fuerzas Armas y la defensa contribuyen a la promoción de la marca España en el exterior.

\section{LA INCORPORACIÓN DE LA MUJER A LAS FAS COMO MODELO DE INTEGRACIÓN}

La incorporación de la mujer en las FAS en España ha sido un proceso que se podría clasificar como tardío en el tiempo ya que se inició en 1988 pero muy 
rápido en su progresión. En poco más de una década, se ha conseguido establecer una legislación escalonada que ha permitido alcanzar la total igualdad a todos los niveles. Desde 1999, la mujer tiene libre acceso a cualquier escala y a cualquier puesto, incluido los de combate y los submarinos. Para valorar este progreso, simplemente basta aportar dos datos: en EEUU la prohibición de acceso de la mujer a los submarinos terminó en 2010 y en Gran Bretaña en 2012. Recientemente, EEUU ha anunciado también el levantamiento de la prohibición que tenía la mujer militar para acceder a puestos de combate ${ }^{2}$.

El modelo establecido en España puede considerarse todo un ejemplo a seguir por otros países. No se impusieron cuotas, ni cuerpos especiales para las mujeres, ni pruebas diferentes para el acceso a la profesión militar. A fecha de hoy, la integración es todo un hecho: un $12 \%$ de los efectivos de nuestras FAS son mujeres. Junto con Francia, en donde el porcentaje asciende al $15 \%$, son los dos países con un mayor número de mujeres militares a nivel europeo. Cabe mencionar que, por ejemplo, en Italia, tal presencia es de sólo el 3\%.

Aparte de haber sido un modelo de integración ejemplar, hay otro aspecto que merece la pena resaltar por su relevancia y es el gran valor que las mujeres militares españolas han aportado a las misiones internacionales. En este sentido la participación se sitúa muy por encima de la media europea, con un $9 \%$ frente al $4 \%$ en Europa ${ }^{3}$.

La resolución 1325 de la ONU "mujer, paz y seguridad" promueve la participación de mujeres militares para afrontar las cuestiones de generó en los conflictos en todas sus vertientes. Desde el punto de vista militar es necesario introducir la perspectiva de género en todas las fases de la operación: planeamiento, ejecución y desarrollo.

Los factores culturales y religiosos presentes en muchas zonas en donde se encuentran desplegadas nuestras FAS, hacen que el éxito de la misión dependa, en gran medida del acercamiento a la población civil como forma de ganarse la confianza. No hay mejor ejemplo, que el de Afganistán, en donde para la mujer afgana está prohibido entablar cualquier tipo de comunicación con un hombre que no está incluido dentro de su ámbito familiar, lo que incluye incluso, la atención médica.

La participación de la mujer militar española en misiones como la de ISAF (International Security Assisstance Force) en Afganistán se ha convertido en uno de los elementos clave en términos de eficacia ${ }^{4}$. La posibilidad de interaccionar con mujeres afganas ha supuesto importantes avances en la labor de los PRT (Provincial Reconstruction Teams) además de la obtención de información que ha redundado en una mayor seguridad de las tropas en tareas de contrainsurgencia. Además, España está desarrollando un programa encuadrado dentro de los "Female Engagement team (FET)" siguiendo el ejemplo de EEUU y Canadá. Estos grupos están formados exclusivamente por mujeres militares destinados a establecer contacto con la población local para organizar proyectos de desarrollo educativo y sanitario.

Dentro de Europa, Holanda y España son, en la actualidad líderes en formación de asesores en esta materia tal y como quedó demostrado en el I Curso Internacional de Asesoría de género que tuvo lugar en Madrid en junio de 2012. Esta experiencia y el interés mostrado van a conducir a España a tener una participación muy activa en el establecimiento de una doctrina uniforme a todos los aliados relativa a la cuestión de género en las misiones en el exterior que está siendo impulsada por la OTAN.

\section{LA PARTICIPACIÓN DE LAS FAS EN MISIONES EN EL EXTERIOR}

La participación militar española en misiones internacionales comenzó en 1989. Desde entonces más de 133000 soldados españoles han sido desplegados en el exterior con un balance en víctimas de 167 muertos (36 por acción hostil y 131 por accidentes o enfermedad $)^{5}$.

Esta participación forma parte del compromiso de España con la paz y la legalidad internacional. Así, en el artículo 5 de la Ley de Defensa Nacional/2005 establece que "Ias fuerzas armadas contribuyen militarmente a la seguridad y defensa de España y de sus aliados, en el marco de las organizaciones internacionales de las que España forma parte, así como al mantenimiento de la paz, la estabilidad y la ayuda humanitaria". Por otro lado, y en el artículo 19 de la misma ley hace referencia a las condiciones que se deben dar para las FAS emprendan una misión en el exterior.

a. Que se realicen por petición expresa del Gobierno del Estado en cuyo territorio se desarrollen o estén autorizadas en Resoluciones del Consejo de Seguridad de las Naciones Unidas o acordadas, en su caso, por organizaciones internacionales de las que España forme parte, particularmente la Unión Europea o la Organización del Tratado del Atlántico Norte (OTAN), en el marco de sus respectivas competencias. 
b.Que cumplan con los fines defensivos, humanitarios, de estabilización o de mantenimiento y preservación de la paz, previstos y ordenados por las mencionadas organizaciones.

c. Que sean conformes con la Carta de las Naciones Unidas y que no contradigan o vulneren los principios del derecho internacional convencional que España ha incorporado a su ordenamiento, de conformidad con el artículo 96.1 de la Constitución.

El cumplimiento de estas condiciones supone para España asumir el ejercicio de un compromiso colectivo internacional. No cabe desvincular la participación de nuestras FAS en misiones internacionales de una acción de política exterior de nuestro país. España debe demostrar que es un socio fiable, responsable y comprometido con la seguridad global en donde las amenazas cada vez son más inciertas y es prácticamente imposible que ninguna nación las pueda abordar en solitario.

Hacer balance de la participación española en el exterior, es poner de manifiesto el excelente papel desempeñado y reconocido internacionalmente por nuestro militares. Y qué mejor ejemplo que el renombramiento de la Plaza Hit como Plaza España en Mostar en reconocimiento de la labor realizado por las tropas españolas en la misión de Bosnia-Herzegovina. Según palabras de la ex embajadora Bosnia el día de la inauguración celebrada el 3 de abril de 2012, la justificación del nuevo nombre de la Plaza se debe a que "La población de Mostar aprecia el sacrifico, esfuerzo y respeto que los soldados españoles demostraron durante la época más dura que vivimos. Por ello todos nos pusimos de acuerdo, justo después del conflicto para poner el nombre de "Plaza de España" a ese lugar de conflicto. Es la plaza más bonita y la plaza mayor de Mostar. Algo que siempre nos unirá como hermanos a los ciudadanos de Mostar, España y Bosnia Herzegovina".

En relación a las misiones en 2013 , ha habido presencia militar en los siguientes escenarios:

\begin{tabular}{|c|c|c|c|c|c|}
\hline $\begin{array}{l}\text { NOMBRE DE LA } \\
\text { MISIÓN }\end{array}$ & $\begin{array}{l}\text { LUGAR } \\
\text { GEOGRÁFICO }\end{array}$ & $\begin{array}{c}\text { Año de } \\
\text { inicio de la } \\
\text { participación } \\
\text { española }\end{array}$ & ORGANIZACIÓN & OBJETIVO & № DE EFECTIVOSI \\
\hline ISAF & Afganistán & 2001 & OTAN & $\begin{array}{l}\text { Reconstrucción y } \\
\text { adiestramiento }\end{array}$ & 1300 \\
\hline EUFOR ALTHEA & Bosnia-Herzegovina & 2004 & UE & $\begin{array}{l}\text { Adiestramiento y } \\
\text { asesoramiento }\end{array}$ & 11 \\
\hline UNIFIL & Líbano & 2006 & ONU & $\begin{array}{l}\text { Vigilancia cese de } \\
\text { hostilidades }\end{array}$ & 700 \\
\hline ATALANTA & Océano Índico & 2008 & UE & Luchar contra la piratería. & 300 \\
\hline EUTM SOMALIA & Uganda y Somalia & 2010 & UE & $\begin{array}{l}\text { Adiestramiento y } \\
\text { asesoramiento fuerzas de } \\
\text { seguridad somalíes }\end{array}$ & 5 \\
\hline EURCAP NÉSTOR & $\begin{array}{l}\text { Djibouti, Kenia y } \\
\text { Sheychelles }\end{array}$ & 2012 & UE & $\begin{array}{l}\text { Formación y capacitación } \\
\text { en la mar }\end{array}$ & $\begin{array}{l}2 \text { militares y un } \\
\text { civil }\end{array}$ \\
\hline EUTM (Malí) & Mali & 2013 & UE & $\begin{array}{l}\text { Adiestramiento y } \\
\text { asesoramiento }\end{array}$ & 24 \\
\hline
\end{tabular}


La participación en estas misiones, ha supuesto para España tener un reconocimiento internacional. En lo que se refiere a la misión en Afganistán, un motivo de orgullo lo encontramos en la labor que se está realizando en la provincia de Badghis, que junto con Herat son las dos provincias en donde se encontraban desplegados los militares españoles. En Badghis es donde se ha registrado el mayor número de reinserciones de insurgentes con un total de 1395. Además, el traspaso de la responsabilidad de la seguridad de la zona a las fuerzas afganas se ha realizado conforme al calendario previsto. A este cumplimiento de objetivos hay que añadir la finalización del tramo correspondiente a la ruta Lithium y que está bajo la seguridad de tropas españolas entre las poblaciones de Qala-i-Naw y Mangan.

La ruta Lithium, también es conocida como "ruta de la luz", tiene un alto valor estratégico ya que con ella se pretende unir Qala-i-Naw con la ciudad de Bala Murghab ciudad que se adentra en el valle de Murghab considerado como guarida de los talibanes y principal vía para traspasar opio a la vecino Turkmenistán. El tramo ejecutado por las tropas españolas, se ha realizado mediante el establecimiento de un equipo de reconstrucción provincial (PRT) que integra un componente civil y otro militar. El tramo de carretera cuya construcción era responsabilidad de España tiene una longitud de $107 \mathrm{Km}$ y ha supuesto un coste de 3 millones de euros financiados por la AECID (Agencia Española de Cooperación Internacional para el Desarrollo). Concluida la parte española, la ruta quedará terminada cuando se realice el tramo norte a cargo de las tropas italianas.

En el diseño del proceso de reconstrucción de Afganistán, el gobierno afgano ha puesto en marcha un "Plan de Infraestructuras Críticas" necesarias para el desarrollo del país. El plan contempla la finalización de una autopista circular, la conocida como "Ring Road" y la construcción de aeropuertos internacionales en Kabul, Kandahar, Herat y Maza-el-Sharif. En esta fase las empresas españolas del sector pueden encontrar una oportunidad para participar en la realización de los proyectos en unas condiciones de seguridad garantizadas por las autoridades afganas.

Otro ejemplo, de lo que aportan las fuerzas armadas a la proyección de la imagen de España en el exterior, es la actuación española en la operación Atalanta. Esta operación, que se inició en 2008, es la primera misión aeronaval que realiza la UE y en ella, España está llevando a cabo una participación muy activa y exitosa. Junto con Francia son las dos naciones que aportan mayor número de naves, con dos y cuatro, respectivamente, les siguen Alemania, Portugal, Suecia y Países Bajos con un buque cada uno. España ha liderado en cuatro ocasiones el Mando de la Fuerza, la última de ellas finalizada en abril de 2013.

En 2012 los ataques piratas se redujeron en un $80 \%$.No obstante, y aunque se hable de éxito habrá que seguir protegiendo a los buques comerciales, y en concreto a los pesqueros. Dados los intereses nacionales de la zona, y a los recortes en el gasto militar que están llevando a cabo en Europa, se corre el riesgo de que la fuerza naval la constituyan cada vez menos países. Quizás entonces, España y Francia, los principales interesados, se queden como los dos actores protagonistas comprometidos con la seguridad de la navegación en el Océano Índico.

Y no se podría concluir el apartado de misiones internacionales de nuestras FAS, sin menciona a la misión en el Líbano (UNIFIL). El contingente español se encuentra en repartido en dos sectores: el este del Líbano y la ciudad de Naqoura en la costa. En el sector este, los militares españoles ejercen su misión en colaboración con oficiales, suboficiales y soldados de Malasia, Nepal, India, Indonesia, Chin, Serbia y El Salvador. Diferentes, lenguas, culturas y religiones que no suponen ningún impedimento para que nuestros militares desarrollen sus actividades, entre las que se incluyen las denominadas CIMIC (Coordinación cívico-militar), como el desminado, apoyo sanitario, reconstrucción de infraestructuras y clases de español. El objetivo de las mismas es el establecimiento de vínculos con la población que repercute en la seguridad de los militares. En este sentido, hay que destacar el "Proyecto Cervantes" que consiste en la enseñanza del castellano a la población local. Son los propios militares los que hacen de profesores en su tiempo libre, apoyados por el material didáctico que cede el Instituto.

Estos tres ejemplos: ISAF, ATALANTA y LÍBANO descritos someramente en este apartado constituyen una clara muestra de la proyección internacional de nuestras Fuerzas Armadas y de su claro papel como embajadoras de la Marca España. Su huella de participación está marcada por el compromiso, su profesionalidad, fiabilidad y su capacidad de entendimiento en un entorno internacional.

\section{LA INDUSTRIA DE DEFENSA}

Uno de los impulsos económicos que necesita nuestro país para salir de la crisis consiste en la ex- 
ternalización de nuestras empresas. Y la industria de defensa no es ajena a esta necesidad casi impuesta. Los continuos recortes en los gastos de Defensa que están realizando la mayoría de los países occidentales, está empujando a los gobiernos integrados en organizaciones, como la UE o la OTAN a estudiar políticas para compartir capacidades entre sus países miembros y desarrollar programas conjuntos. Dentro de Europa, el gasto en defensa se ha reducido entorno al $10 \%$ y comienzan a surgir propuestas para racionalizar el gasto sin perder capacidades defensivas. Así se habla del término "pooling and sharing" que consiste en establecer una defensa más eficiente mediante la colaboración en el desarrollo de programas bajo los auspicios de la PCSD (Política Común de Seguridad y Defensa), con el apoyo de la EDA (European Defence Agency) y la posibilidad de compartir capacidades entre los países miembros respetando siembre el principio de soberanía nacional.

Esta tendencia pone de manifiesto que las empresas de defensa españolas tendrán que realizar esfuerzos para participar de forma activa en los proyectos que se desarrollen dentro de esos programas colaborativos y a lanzarse a la búsqueda de nuevos mercados emergentes. Y se habla de mercados emergentes, porque así como en la mayoría de los países europeos se ha reducido el gasto militar en un $10 \%$ como ya se ha señalado, en Asia y Oceanía aumentó un 3.3\%, en Oriente Medio un $8.4 \%$ y en América latina un 4.2\%.

Para la industria de defensa de España este cambio supone todo un reto. En primer lugar, porque el sector de la defensa ha gozado de un proteccionismo por parte del gobierno al considerarse un sector relacionado, en cierta medida, con la soberanía nacional lo que ha propiciado que el Ministerio de Defensa y las propias empresas hayan realizado grandes inversiones. Este apoyo recibido al desarrollo nacional de sistemas y tecnologías es difícil que encuentre un retorno económico en el mercado nacional. En segundo lugar, porque la competitividad de las empresas de defensa pasa por buscar nuevos modelos productivos en el exterior, y en tercer lugar, porque la apertura a nuevos mercados tiene que ir acompañada de un apoyo comercial ejercido por el propio gobierno en el marco de acuerdos bilaterales con otros organismos gubernamentales del país cliente.

Pero estos retos, suponen a la vez, nuevas oportunidades de lanzar al exterior una industria de defensa española de elevado nivel tecnológico capaz de competir en los mercados internacionales y convertirse en uno de las principales vías de promoción de la "Marca España". Limitado el mercado interior y la posibilidad futura de avanzar hacia unas capacidades comparti- das con nuestros socios y aliados, obliga a la industria nacional a aumentar su presencia internacional.

Durante las últimas décadas a la industria de defensa española ha alcanzado un nivel de capacitación e innovación tecnológica muy notable gracias a los programas que se han ido impulsando desde el Ministerio de Defensa. Pero la crisis económica y los recortes llevados a cabo por el Ministerio han provocado una contracción del mercado interior, que se sitúa en torno a 47 millones de euros en 2011 con respecto al 2010. Sin embargo, en el mismo periodo las exportaciones se han incrementado en torno al 6.8\% (aproximadamente unos 252 millones de euros). Hay otro dato que merece la pena destacar como es la concentración empresarial del sector en el negocio internacional. El 90\% de las ventas en 2011 se atribuyen a cinco empresas, EADS-CASA, NAVANTIA, AIRBUS MILITARY, INDRA SISTEMAS, e INDUSTRIA DE TURBOPROPULSORES. EI 10\% restante queda repartido entre 75 empresas. En cuanto a los sectores, el que mayor cifra de negocio obtuvo en 2011 fue el aeronáutico con unas ventas de 2889 millones de euros, seguido del naval con 553 millones de euros.

El Ministerio de Defensa, tomando conciencia de la dificultad con la que se pueden encontrar nuestras grandes empresas de defensa para penetrar en otros mercados, ha decidido llevar a cabo una política de lanzamiento al exterior. La directiva de Defensa Nacional 2012 es clara a este respecto: “...la defensa de España exige el impulso de la industria nacional del sector, suministrador idóneo de las necesidades de nuestras Fuerzas Armadas. En los tiempos actuales, la permanencia y desarrollo de la misma hace precisa la asistencia a esta en su presencia internacional, especialmente en la penetración en mercados que por su especificidad observan garantías mayores con el respaldo de Estado a Estado".

En la misma línea hay que mencionar la aprobación del Real Decreto 19/2012 del 25 de mayo que permite al Ministerio de Defensa tener una participación más activa en la gestión de programas dirigidos a la exportación poniendo fin a una clara situación de desventaja en la que se encontraban las empresas nacionales frente a las de otros países.

Salvado este escollo legislativo, no es casualidad, por tanto que en la agenda de los viajes del Ministro de Defensa, Pedro Morenés, esté incluida la promoción y el apoyo de la industria nacional de defensa. $Y$ se pueden poner como ejemplos, los viajes a Chile, Perú, Brasil, Australia, Indonesia, Cabo Verde, Polonia, Argelia, Arabia Saudí y Emiratos Árabes Unidos. En Brasil, se trató la posible adquisición de aviones de transporte militar C-295 y la modernización de 
los aviones P-3 Orion. En Indonesia, el ministro ofreció excedente de material de las FAS y la posibilidad de cooperación en el ámbito naval y aéreo como el acuerdo conseguido por Indra, que roza los 10 millones de euros, para participar en la modernización de tres de sus submarinos y la venta de los sistemas radar para controlar el 70\% del espacio aéreo Indonesio.

En Australia, Navantia ha encontrado un gran mercado. En febrero de 2013, coincidiendo con la visita del ministro se produjo la puesta en servicio del buque de proyección estratégica "El Camberra", el primero de los dos contratados y fabricados por Navantia. Además, la cesión del buque español "Cantabria" durante un año al gobierno australiano es la mejor forma de que conozcan las características del barco con vistas a futuros contratos. Y en Arabia Saudí en 2012, el ministro apoyó la venta de carros de combate Leopard por un importe de 3000 millones, y la adquisición de vehículos URO. En este mismo país, aprovechando que la fragata Méndez Núnez estaba regresando de la operación Atalanta, atracó en el puerto de Jeddah donde fue recibida por una delegación de la Marina Saudí y el Presidente de Navantia. Y para concluir esta lista de ejemplos, el Secretario de Estado de Defensa, el Ministro de Defensa, Pedro Argüelles, encabezó la delegación española asistentes a la Feria Internacional de Defensa y Seguridad (LADD) celebrada en Brasil en el mes de abril de 2013. Brasil está llevando a cabo una política para impulsar la modernización de sus FAS por lo que su mercado está considerado de un alto valor estratégico. La experiencia y el desarrollo tecnológico aportado por las empresas españolas pueden constituir una gran baza para hacerse con un trozo del pastel. Eso es la "Marca España”.
1 En el IX estudio CIS titulado "FUERZAS ARMADAS Y SOCIEDAD". Un 42,8\% de la población entrevistada considera que las Fas contribuyen "bastante" al prestigio de un país mientras que un $18,8 \%$ opina que contribuye "mucho".

2 El 23 de enero de 2013, el Secretario de Defensa de EEUU, Leon Panneta, anun- ció el fin de la prohibición del acceso de la mujer a los puestos de combate.

3 Dato obtenido en el I Curso Internacional de Asesoría de Género en Operaciones celebrado el 11 de junio de 2012 en Madrid.
4 Declaraciones de la Subsecretaria de Estado de Defensa, Irene, Domínguez-Alcahud en la inauguración del I Curso Internacional de Asesoría de Género en Operaciones celebrado el 11 de junio de 2012.

5 A fecha 16 de marzo de 2013.

\section{BIBLIOGRAFÍA}

Informe del IEEE. "Estudio encuesta "Defensa Nacional y Fuerzas Armadas". Octubre 2011. Disponible en:http:// www.ieee.es/Galerias/fichero/ESociologicos/2011/INFORME_CIS_2011_COMPLETO_y_ACTUALIZADO-.pdf

Real Decreto Ley 1/1988, de 12 de febrero, por el que se regula la incorporación de la mujer a las Fuerzas Armadas.

Ley $17 / 1989$, de 19 de julio, reguladora del Régimen del Personal Militar Profesional (vigente hasta el 20 de mayo de 1999).

Real Decreto 984/1992, de 31 de julio, por el que se aprueba el Reglamento de Tropa y Marinería profesional de las Fuerzas Armadas.

Ley $17 / 1999$, de 18 de mayo, de régimen del Personal de las FAS.
Real Decreto 66/2000, de 21 de enero, por el que se modifican las condiciones de acceso a militar profesional de Tropa y Marinería de las Fueras Armadas.

http://www.defensa.gob.es/Galerias/ areasTematicas/observatorio/fichero/ estadisticas/Estadisticas-Observatoriodiciembre-2012.pdf

http://www.mujer.info/espana-es-uno-delos-paises-europeos-con-mas-mujeresmilitares/

http://www.defensa.gob.es/areasTematicas/misiones/. Consultada 16 de marzo de 2013.

http://www.abc.es/20120329/espana/ abci-huella-militar-espana-mostar-201203281626.html
Montánchez, Enrique. "Ruta Lithium, misión cumplida". Revista Española de Defensa. Septiembre 2012.

http://eunavfor.eu/deployed-units/surfacevessels/ consultada el 12 de abril de 2013

YearBook 2012, SIPRI (Stockholm International Peace Research Institute)

Informe- 2011. "La industria de Defensa en España". Dirección General de Armamento y Material. Ministerio de Defensa

http://economía.elpais.com/economía / 2013 / 03 / 15 / a gen cias/1363361749_497488.html

http://www.revistatenea.es/revistaatenea/revista/articulos/GestionNoticias_11993_ESP.asp 